\title{
POINT-SPLITTING REGULARIZATION FOR GAUGE THEORIES: QUANTUM ELECTRODYNAMICS
}

\author{
Conrad Newton* \\ Gordon McKay Laboratory, Harvard University \\ Cambridge, Massachusetts 02138, U.S.A. \\ and \\ Theoretical Physics Division, CERN \\ CH 1211 Geneva 23, Switzerland \\ Per Osland $§$ \\ Department of Physics, University of Bergen, Allégt. 55 \\ N-5007 Bergen, Norway \\ and \\ Tai Tsun $\mathrm{Wu}^{*}$ \\ Gordon McKay Laboratory, Harvard University \\ Cambridge, Massachusetts 02138, U.S.A. \\ and \\ Theoretical Physics Division, CERN \\ CH 1211 Geneva 23, Switzerland
}

\begin{abstract}
As a method of regularization, point splitting has played an essential role in the recent theoretical determination of the masses of the Higgs boson and the top quark. It is the purpose of this paper to put this point-splitting regularization on a firm basis. The result turns out to be extremely simple: replace the usual vertex factor $-i e \gamma_{\mu}$ in quantum electrodynamics by

$$
-i e\left(\gamma_{\mu}-\frac{\not p}{p \cdot \delta} \delta_{\mu}\right)
$$

where $p$ is the momentum of the photon line, and $\delta_{\mu}$ is the distance for point splitting. No additional vertices are needed.

* Work supported in part by the U.S. Department of Energy under Grant DE-FG0284ER40158.

$\S$ Work supported in part by the Research Council of Norway (NAVF). CERN-TH.7007/93
\end{abstract}

September 1993 


\section{Introduction}

Recent theoretical studies [1], [2], [3] of the standard model [4] indicate that the masses of the as yet unobserved Higgs boson [5] and the top quark are given by

$$
\begin{aligned}
m_{H}^{2} & =9 m_{Z}^{2}-6 m_{W}^{2} \\
m_{t}^{2} & =\frac{5}{2} m_{Z}^{2}-m_{W}^{2}
\end{aligned}
$$

where the masses of the $b$ quark and lighter fermions are neglected. In this development, point splitting [6] is used as the method of regularization.

These relations (1.1) are not exact; there are radiative corrections due to two-loop diagrams [7]. For these radiative corrections, the point-splitting regularization of [1] is not adequate. Therefore a more systematic development for such regularization is needed; it is the purpose of this paper to supply this development for the simplest case of quantum electrodynamics.

For the purpose of orientation, the following two comments may be useful:

(1) Point splitting for gauge theories is a much richer subject than for quantum field theories without gauge invariance.

(2) There are two distinct approaches to applying point splitting to gauge theories:

(a) Point splitting is applied at only one of the vertices for each Feynman diagram; and

(b) Point splitting is used at every vertex of every Feynman diagram.

As discussed in [1], approach (b) is to be preferred for the present purpose, although approach (a) was used in [6]. This use of (b) can perhaps be considered to be a characteristic of point-splitting regularization.

As already stated in the Abstract, the result for approach (b) is extremely simple for quantum electrodynamics: with point splitting, the Feynman rule for the eer vertex is

$$
-i e\left(\gamma_{\mu}-\frac{\not p}{p \cdot \delta} \delta_{\mu}\right)
$$

where $p$ is the momentum of the photon line, and $\delta_{\mu}$ is the four-vector for point-splitting. This (1.2) is to be compared with the usual Feynman rule of $-i e \gamma_{\mu}$. When (1.2) is used, there are no additional vertices such as ee $\gamma \gamma$, ee $\gamma \gamma \gamma$ etc. These extra vertices, discussed in [1], are due to the presence of the second term in (1.2).

Clearly, when contracted with $p^{\mu}$, this expression (1.2) gives zero. Thus, gauge invariance is assured. 
As a first exercise, the Feynman rule (1.2) is applied to the case of the one-loop photon self-energy in Sec. 2. This exercise shows major similarity, but also important differences, from the corresponding treatment in [1]. The differences are studied in detail in Sec. 3, where we also show that the present result and the previous one for the one-loop photon self-energy are actually identical.

The rest of this paper is devoted to answering the following two important questions: (A) How should the singularity in (1.2) due to the vanishing of $(p \cdot \delta)$ be interpreted?

(B) Is point-splitting regularization for quantum electrodynamics unambiguous?

Actually, question (A) can be considered to be a part of question (B), which has an affirmative answer for any given $\delta$.

\section{Photon self-energy}

Let the vertex (1.2) be applied to the one-loop photon self-energy diagram of Fig. 1 [1]:

$$
\begin{aligned}
I_{\mu \nu}^{T}= & \frac{-1}{(2 \pi)^{4}} \int \mathrm{d}^{4} k e^{2 i k \cdot \delta} \\
\times & \operatorname{Tr}\left[\frac{i}{\not k+\frac{1}{2} \not p-m+i \epsilon}(-i e)\left(\gamma_{\mu}-\frac{\not p}{p \cdot \delta} \delta_{\mu}\right)\right. \\
& \left.\times \frac{i}{\not k-\frac{1}{2} \not p-m+i \epsilon}(-i e)\left(\gamma_{\nu}-\frac{\not p}{p \cdot \delta} \delta_{\nu}\right)\right] .
\end{aligned}
$$

Let $I_{\mu \nu}$ be the corresponding formula without the second term of (1.2), i.e.,

$$
\begin{aligned}
I_{\mu \nu} & =\frac{-1}{(2 \pi)^{4}} \int \mathrm{d}^{4} k e^{2 i k \cdot \delta} \\
& \times \operatorname{Tr}\left[\frac{i}{\not k+\frac{1}{2} \not p-m+i \epsilon}\left(-i e \gamma_{\mu}\right) \frac{i}{\not k-\frac{1}{2} \not p-m+i \epsilon}\left(-i e \gamma_{\nu}\right)\right] .
\end{aligned}
$$

This $I_{\mu \nu}$ is the same as that of eq. (3.3) of [1].

Let $I_{\mu \nu}^{\prime}$ be the difference

$$
I_{\mu \nu}^{\prime}=I_{\mu \nu}^{T}-I_{\mu \nu},
$$

then

$$
\begin{aligned}
I_{\mu \nu}^{\prime} & =\frac{-1}{(2 \pi)^{4}} \int \mathrm{d}^{4} k e^{2 i k \cdot \delta}\left[-(-i e)^{2}\right] \\
& \times \operatorname{Tr}\left[\frac{i}{\not k+\frac{1}{2} \not p-m+i \epsilon} \frac{\not p}{p \cdot \delta} \frac{i}{\not k-\frac{1}{2} \not p-m+i \epsilon}\left(\delta_{\mu} \gamma_{\nu}+\gamma_{\mu} \delta_{\nu}-\frac{\not p}{p \cdot \delta} \delta_{\mu} \delta_{\nu}\right)\right] .
\end{aligned}
$$


By the standard maneuver of using

$$
\not p=\left(\not k+\frac{1}{2} \not p-m+i \epsilon\right)-\left(\not k-\frac{1}{2} \not p-m+i \epsilon\right),
$$

equation (2.4) can be rewritten as

$$
\begin{aligned}
I_{\mu \nu}^{\prime} & =\frac{-1}{(2 \pi)^{4}} \int \mathrm{d}^{4} k e^{2 i k \cdot \delta}\left[-(-i e)^{2}\right] \\
& \times \operatorname{Tr}\left[\left(\frac{i}{\not k-\frac{1}{2} \not p-m+i \epsilon}-\frac{i}{\not k+\frac{1}{2} \not p-m+i \epsilon}\right) \frac{i}{p \cdot \delta}\left(\delta_{\mu} \gamma_{\nu}+\gamma_{\mu} \delta_{\nu}-\frac{\not p}{p \cdot \delta} \delta_{\mu} \delta_{\nu}\right)\right](2.6)
\end{aligned}
$$

Because of the point-splitting regularization, it is allowed to shift the variable of integration, $k$, by $\pm \frac{1}{2} p$ to get

$$
\begin{aligned}
I_{\mu \nu}^{\prime} & =\frac{-1}{(2 \pi)^{4}} \int \mathrm{d}^{4} k e^{2 i k \cdot \delta} \\
& \times \operatorname{Tr}\left[\frac{i}{\not k-m+i \epsilon}\left(-2 e^{2}\right) \frac{\sin (p \cdot \delta)}{p \cdot \delta}\left(\delta_{\mu} \gamma_{\nu}+\gamma_{\mu} \delta_{\nu}-\frac{\not p}{p \cdot \delta} \delta_{\mu} \delta_{\nu}\right)\right] .
\end{aligned}
$$

With the vertex (1.2), this is the extra piece due to the point splitting, i.e., due to the second term $-\not p \delta_{\mu} /(p \cdot \delta)$.

\section{Comparison with the Feynman rules used previously}

In [1], the additional contribution to the one-loop photon self-energy besides $I_{\mu \nu}$ is interpreted in terms of a peculiar diagram with an ee $\gamma \gamma$ vertex. From equation (2.7), the Feynman rule for this $e e \gamma \gamma$ vertex is seen to be

$$
V_{\mu \nu}^{(2)}(p,-p)=-2 e^{2} \frac{\sin (p \cdot \delta)}{p \cdot \delta}\left(\delta_{\mu} \gamma_{\nu}+\gamma_{\mu} \delta_{\nu}-\frac{\not p}{p \cdot \delta} \delta_{\mu} \delta_{\nu}\right)
$$

This is to be compared with the Feynman rule used previously [1]

$$
V_{\mu \nu}^{(2) \prime}(p,-p)=-e^{2} \frac{\sin (p \cdot \delta)}{p \cdot \delta}\left(\delta_{\mu} \gamma_{\nu}+\gamma_{\mu} \delta_{\nu}\right)
$$

It is seen that they are quite different: the $V_{\mu \nu}^{(2)}(p,-p)$ of eq. (3.1) has an extra term and also an extra overall factor of 2 .

It is nevertheless true that the $I_{\mu \nu}^{\prime}$ of Sec. 2 here is the same as the $I_{\mu \nu}^{\prime}$ of ref. [1]. To see this, the important point is that, by Lorentz invariance,

$$
\int \mathrm{d}^{4} k e^{2 i k \cdot \delta} \frac{k_{\mu}}{k^{2}-m^{2}+i \epsilon}=\operatorname{constant} \cdot \delta_{\mu} .
$$


Therefore, so far as this one-loop photon self-energy is concerned, it is permissible to use the substitution

$$
k_{\mu} \rightarrow c_{0} \delta_{\mu}
$$

in the numerator of the integrand. If (3.2) is used, the numerator is

$$
\begin{aligned}
-e^{2} & \frac{\sin (p \cdot \delta)}{p \cdot \delta}\left(\delta_{\mu} k_{\nu}+k_{\mu} \delta_{\nu}\right) \rightarrow-e^{2} \frac{\sin (p \cdot \delta)}{p \cdot \delta} c_{0}\left(\delta_{\mu} \delta_{\nu}+\delta_{\mu} \delta_{\nu}\right) \\
= & -2 e^{2} \frac{\sin (p \cdot \delta)}{p \cdot \delta} c_{0} \delta_{\mu} \delta_{\nu} .
\end{aligned}
$$

On the other hand, if (3.1) is used instead, the numerator is

$$
\begin{aligned}
-2 e^{2} & \frac{\sin (p \cdot \delta)}{p \cdot \delta}\left(\delta_{\mu} k_{\nu}+k_{\mu} \delta_{\nu}-\frac{p \cdot k}{p \cdot \delta} \delta_{\mu} \delta_{\nu}\right) \\
& \rightarrow-2 e^{2} \frac{\sin (p \cdot \delta)}{p \cdot \delta} c_{0}\left(\delta_{\mu} \delta_{\nu}+\delta_{\mu} \delta_{\nu}-\frac{p \cdot \delta}{p \cdot \delta} \delta_{\mu} \delta_{\nu}\right) \\
& =-2 e^{2} \frac{\sin (p \cdot \delta)}{p \cdot \delta} c_{0} \delta_{\mu} \delta_{\nu} .
\end{aligned}
$$

Since the right-hand sides of (3.5) and (3.6) are the same, the two $I_{\mu \nu}^{\prime}$ are identical.

That these two $I_{\mu \nu}^{\prime}$ are the same has the following implication. With the previous Feynman rule (3.2), there is clearly no singularity at $p \cdot \delta=0$. Therefore the presence of $p \cdot \delta$ in the denominator of (1.2) does not lead to any such singularity for the one-loop photon self-energy. In other words, for the diagram of Fig. 1, it is permissible to interpret this $(p \cdot \delta)^{-1}$ in any way one wishes so long as the same interpretation is used for every occurrence of $(p \cdot \delta)^{-1}$. It will be seen in Sec. 4 that this is not true for more complicated diagrams.

Since the expressions (3.1) and (3.2) are in general significantly different, it is desirable to have a derivation of the Feynman rule (3.1) from first principles. This will be carried out in the remainder of this section.

The derivation is based on the standard diagrammatic verification of gauge invariance in quantum electrodynamics. Consider first the diagram of Fig. 2 (a), with the choice of momenta as indicated. In this case, the exponential factor due to point splitting is

$$
\begin{aligned}
& \exp \left(\frac{1}{2} i\left\{\left[k-\frac{1}{2}\left(p_{1}+p_{2}\right)\right] \cdot \delta+2\left[k+\frac{1}{2}\left(p_{1}-p_{2}\right)\right] \cdot \delta+\left[k+\frac{1}{2}\left(p_{1}+p_{2}\right)\right] \cdot \delta\right\}\right) \\
& =e^{2 i k \cdot \delta} e^{\frac{i}{2}\left(p_{1}-p_{2}\right) \cdot \delta} .
\end{aligned}
$$

Omitting the first factor $e^{2 i k \cdot \delta}$ and contracting the amplitude corresponding to the diagram of fig. 2 (a) with $p_{1 \mu}$ we get

$$
e^{\frac{i}{2}\left(p_{1}-p_{2}\right) \cdot \delta}\left(-i e \gamma_{\nu}\right) \frac{i}{\not k+\frac{1}{2}\left(\not p_{1}-\not p_{2}\right)-m+i \epsilon}\left(-i e p_{1}\right) .
$$


As in equation (2.5), $\not p_{1}$ can be written as

$$
\not p_{1}=\left[\not k+\frac{1}{2}\left(\not p_{1}-\not p_{2}\right)-m+i \epsilon\right]-\left[\not k-\frac{1}{2}\left(\not \not_{1}+\not p_{2}\right)-m+i \epsilon\right]
$$

When only the first term on the right-hand side of (3.9) is retained, (3.8) leads to

$$
-i e^{2} e^{\frac{i}{2}\left(p_{1}-p_{2}\right) \cdot \delta} \gamma_{\nu}
$$

When the same procedure is applied to the diagram of Fig. 2 (b) except that only the second term in the analog of (3.9) (i.e., with $p_{2} \rightarrow-p_{2}$ ) is retained, the result is

$$
i e^{2} e^{-\frac{i}{2}\left(p_{1}-p_{2}\right) \cdot \delta} \gamma_{\nu}
$$

In the absence of point splitting, i.e., when $\delta=0$, the verification of gauge invariance follows from the vanishing of the sum of (3.10) and (3.11). When $\delta$ is not zero, the sum of (3.10) and (3.11) is

$$
2 e^{2} \gamma_{\nu} \sin \left[\frac{1}{2}\left(p_{1}-p_{2}\right) \cdot \delta\right]
$$

which is no longer zero.

In order to recover gauge invariance, an $e e \gamma \gamma$ vertex must be introduced to cancel this (3.12). Let $V_{\mu \nu}^{(2)}\left(p_{1}, p_{2}\right)$ be the Feynman rule for this $e e \gamma \gamma$ vertex, where $p_{1}$ and $p_{2}$ are the incoming momenta of the two photons. This $V_{\mu \nu}^{(2)}\left(p_{1}, p_{2}\right)$ must satisfy

$$
V_{\mu \nu}^{(2)}\left(p_{1}, p_{2}\right)=V_{\nu \mu}^{(2)}\left(p_{2}, p_{1}\right)
$$

and

$$
p_{1 \mu} V_{\mu \nu}^{(2)}\left(p_{1}, p_{2}\right)=-2 e^{2} \gamma_{\nu} \sin \left[\frac{1}{2}\left(p_{1}-p_{2}\right) \cdot \delta\right]+U_{\nu}\left(p_{1}, p_{2}\right) \text {, }
$$

where the function $U_{\nu}\left(p_{1}, p_{2}\right)$ is chosen to be of the form

$$
U_{0 \nu}\left(p_{1}, p_{2}\right)\left(\not p_{1}+\not p_{2}\right)
$$

The reason for the introduction of $U_{\nu}$ in the form (3.15) is as follows. If $U_{\nu}$ is chosen to be zero, then there is no $V_{\mu \nu}^{(2)}\left(p_{1}, p_{2}\right)$ that satisfies both (3.13) and (3.14). The form (3.15) is the only one that allows further cancellation of denominators through

$$
\not p_{1}+\not p_{2}=\left[\not k+\frac{1}{2}\left(\not p_{1}+\not p_{2}\right)-m+i \epsilon\right]-\left[\not k-\frac{1}{2}\left(\not p_{1}+\not p_{2}\right)-m+i \epsilon\right]
$$

(see Fig. 2). 
By (3.13), the most general form for $V_{\mu \nu}^{(2)}\left(p_{1}, p_{2}\right)$ is

$$
\begin{aligned}
V_{\mu \nu}^{(2)}\left(p_{1}, p_{2}\right) & =A_{1}\left(p_{1}, p_{2}\right) \delta_{\mu} \gamma_{\nu}+A_{1}\left(p_{2}, p_{1}\right) \gamma_{\mu} \delta_{\nu} \\
& +\delta_{\mu} \delta_{\nu}\left[\not p_{1} A_{2}\left(p_{1}, p_{2}\right)+\not p_{2} A_{2}\left(p_{2}, p_{1}\right)\right]
\end{aligned}
$$

Substitution into (3.14) then gives

$$
A_{1}\left(p_{1}, p_{2}\right)\left(p_{1} \cdot \delta\right)=-2 e^{2} \sin \left[\frac{1}{2}\left(p_{1}-p_{2}\right) \cdot \delta\right]
$$

and

$$
\begin{aligned}
U_{0 \nu} & =\delta_{\nu}\left[A_{1}\left(p_{2}, p_{1}\right)+\left(p_{1} \cdot \delta\right) A_{2}\left(p_{1}, p_{2}\right)\right] \\
& =\delta_{\nu}\left(p_{1} \cdot \delta\right) A_{2}\left(p_{2}, p_{1}\right) .
\end{aligned}
$$

Equation (3.19) shows explicitly that $U_{0 \nu}$ cannot be zero. Thus,

$$
A_{1}\left(p_{1}, p_{2}\right)=-2 e^{2} \frac{1}{p_{1} \cdot \delta} \sin \left[\frac{1}{2}\left(p_{1}-p_{2}\right) \cdot \delta\right]
$$

and

$$
A_{2}\left(p_{1}, p_{2}\right)-A_{2}\left(p_{2}, p_{1}\right)=-2 e^{2} \frac{1}{\left(p_{1} \cdot \delta\right)\left(p_{2} \cdot \delta\right)} \sin \left[\frac{1}{2}\left(p_{1}-p_{2}\right) \cdot \delta\right],
$$

where the right-hand side does have the correct symmetry property under the interchange of $p_{1}$ and $p_{2}$. Therefore

$$
\begin{gathered}
V_{\mu \nu}^{(2)}\left(p_{1}, p_{2}\right)=-\frac{e^{2}}{\left(p_{1} \cdot \delta\right)\left(p_{2} \cdot \delta\right)}\{ \\
\sin \left[\frac{1}{2}\left(p_{1}-p_{2}\right) \cdot \delta\right]\left[2\left(p_{2} \cdot \delta\right) \delta_{\mu} \gamma_{\nu}-2\left(p_{1} \cdot \delta\right) \gamma_{\mu} \delta_{\nu}+\left(\not p_{1}-\not p_{2}\right) \delta_{\mu} \delta_{\nu}\right] \\
\left.+A\left(p_{1}, p_{2}\right)\left(p_{1}+\not p_{2}\right) \delta_{\mu} \delta_{\nu}\right\}
\end{gathered}
$$

where $A\left(p_{1}, p_{2}\right)$ is an arbitrary symmetric function of $p_{1}$ and $p_{2}$.

When $p_{1}=-p_{2}=p$, eq. (3.22) reduces to (3.1), not to (3.2). This is the derivation of (3.1). With this derivation, the steps given in Sec. 2 can be carried out in reverse, showing that the vertex (1.2) is not only desirable from the point of view of gauge invariance, but is in fact unique.

\section{Three-photon vertex and charge conjugation}

There are two one-loop $\gamma \gamma \gamma$ vertex diagrams, one of which is shown in Fig. 3. The contribution of this diagram is

$$
I_{\mu \nu \sigma}^{T(1)}\left(p_{1}, p_{2}, p_{3}\right)=\frac{-1}{(2 \pi)^{4}} \int \mathrm{d}^{4} k e^{3 i k \cdot \delta}
$$




$$
\begin{aligned}
\times & \operatorname{Tr}\left[\frac{i}{\not k-\frac{1}{3}\left(\not p_{2}-\not p_{3}\right)-m+i \epsilon}(-i \epsilon)\left(\gamma_{\sigma}-\frac{\not p_{3}}{p_{3} \cdot \delta} \delta_{\sigma}\right)\right. \\
& \times \frac{i}{\not k-\frac{1}{3}\left(\not p_{3}-\not p_{1}\right)-m+i \epsilon}(-i \epsilon)\left(\gamma_{\mu}-\frac{\not p_{1}}{p_{1} \cdot \delta} \delta_{\mu}\right) \\
& \left.\times \frac{i}{\not k-\frac{1}{3}\left(\not p_{1}-\not p_{2}\right)-m+i \epsilon}(-i \epsilon)\left(\gamma_{\nu}-\frac{\not p_{2}}{p_{2} \cdot \delta} \delta_{\nu}\right)\right] .
\end{aligned}
$$

Similarly, the contribution of the other diagram, which differs in only having the direction of the electron line reversed, is

$$
\begin{aligned}
I_{\mu \nu \sigma}^{T(2)}\left(p_{1}, p_{2}, p_{3}\right)= & \frac{-1}{(2 \pi)^{4}} \int \mathrm{d}^{4} k e^{3 i k \cdot \delta} \\
\times & \operatorname{Tr}\left[\frac{i}{\not k+\frac{1}{3}\left(\not p_{2}-\not p_{3}\right)-m+i \epsilon}(-i e)\left(\gamma_{\nu}-\frac{\not p_{2}}{p_{2} \cdot \delta} \delta_{\nu}\right)\right. \\
& \times \frac{i}{\not k+\frac{1}{3}\left(\not p_{1}-\not p_{2}\right)-m+i \epsilon}(-i \epsilon)\left(\gamma_{\mu}-\frac{\not p_{1}}{p_{1} \cdot \delta} \delta_{\mu}\right) \\
& \left.\times \frac{i}{\not k+\frac{1}{3}\left(\not p_{3}-\not p_{1}\right)-m+i \epsilon}(-i \epsilon)\left(\gamma_{\sigma}-\frac{\not p_{3}}{p_{3} \cdot \delta} \delta_{\sigma}\right)\right] .
\end{aligned}
$$

Analogous to (2.2), let $I_{\mu \nu \sigma}^{(j)}, j=1,2$ be the corresponding integrals without the second term for the ee $\gamma$ vertex:

$$
\begin{aligned}
I_{\mu \nu \sigma}^{(1)}\left(p_{1}, p_{2}, p_{3}\right)= & \frac{-1}{(2 \pi)^{4}} \int \mathrm{d}^{4} k e^{3 i k \cdot \varepsilon} \\
\times & \operatorname{Tr}\left[\frac{i}{\not k-\frac{1}{3}\left(\not p_{2}-\not p_{3}\right)-m+i \epsilon}\left(-i e \gamma_{\sigma}\right) \frac{i}{\not k-\frac{1}{3}\left(\not p_{3}-\not p_{1}\right)-m+i \epsilon}\left(-i e \gamma_{\mu}\right)\right. \\
& \left.\times \frac{i}{\not k-\frac{1}{3}\left(\not p_{1}-\not p_{2}\right)-m+i \epsilon}\left(-i e \gamma_{\nu}\right)\right]
\end{aligned}
$$

and

$$
\begin{aligned}
I_{\mu \nu \sigma}^{(2)}\left(p_{1}, p_{2}, p_{3}\right)= & \frac{-1}{(2 \pi)^{4}} \int \mathrm{d}^{4} k e^{3 i k \cdot \delta} \\
\times & \operatorname{Tr}\left[\frac{i}{\not k+\frac{1}{3}\left(\not p_{2}-\not p_{3}\right)-m+i \epsilon}\left(-i e \gamma_{\nu}\right) \frac{i}{\not k+\frac{1}{3}\left(\not p_{1}-\not p_{2}\right)-m+i \epsilon}\left(-i e \gamma_{\mu}\right)\right. \\
& \left.\times \frac{i}{\not k+\frac{1}{3}\left(\not p_{3}-\not p_{1}\right)-m+i \epsilon}\left(-i e \gamma_{\sigma}\right)\right]
\end{aligned}
$$

Therefore

$$
I_{\mu \nu \sigma}^{(2)}\left(p_{1}, p_{2}, p_{3}\right)=-\left.I_{\mu \nu \sigma}^{(1)}\left(p_{1}, p_{2}, p_{3}\right)\right|_{\delta \rightarrow-\delta}
$$


Since $I_{\mu \nu \sigma}^{(1)}\left(p_{1}, p_{2}, p_{3}\right)$ is not invariant under $\delta \rightarrow-\delta$, eq. (4.5) implies that in general

$$
I_{\mu \nu \sigma}^{(1)}\left(p_{1}, p_{2}, p_{3}\right)+I_{\mu \nu \sigma}^{(2)}\left(p_{1}, p_{2}, p_{3}\right) \neq 0
$$

Similarly,

$$
I_{\mu \nu \sigma}^{T(1)}\left(p_{1}, p_{2}, p_{3}\right)+I_{\mu \nu \sigma}^{T(2)}\left(p_{1}, p_{2}, p_{3}\right) \neq 0 .
$$

Thus Furry's Theorem [8] is not satisfied by taking into account only these two Feynman diagrams.

This result is to be expected from the fact that, with point splitting, the field $\psi$ is evaluated at $x+\frac{1}{2} \delta$, while the corresponding $\bar{\psi}$ is evaluated at $x-\frac{1}{2} \delta$. Under charge conjugation, $x+\frac{1}{2} \delta$ and $x-\frac{1}{2} \delta$ are interchanged, i.e., $\delta \rightarrow-\delta$. Therefore, in order to recover charge-conjugation invariance, it is necessary to average over $\delta$ and $-\delta$. This averaging is well-known for approach (a) of Sec. 1 [6]; what is shown here is that it is also necessary for the present approach (b).

Thus the sum $I_{\mu \nu \sigma}^{(1)}+I_{\mu \nu \sigma}^{(2)}$ is replaced by

$$
\frac{1}{2}\left[\left(I_{\mu \nu \sigma}^{(1)}+I_{\mu \nu \sigma}^{(2)}\right)+\left.\left(I_{\mu \nu \sigma}^{(1)}+I_{\mu \nu \sigma}^{(2)}\right)\right|_{\delta \rightarrow-\delta}\right],
$$

which is indeed zero by (4.5). With (1.2), we want similarly

$$
\frac{1}{2}\left[\left(I_{\mu \nu \sigma}^{T(1)}+I_{\mu \nu \sigma}^{T(2)}\right)+\left.\left(I_{\mu \nu \sigma}^{T(1)}+I_{\mu \nu \sigma}^{T(2)}\right)\right|_{\delta \rightarrow-\delta}\right]=0 .
$$

While the vanishing of (4.8) follows from (4.3) and (4.4), this (4.9) is not a consequence of (4.1) and (4.2). This subtle point is to be discussed next.

It is shown in Sec. 3 that, although the right-hand side of eq. (2.1) has various powers of $p \cdot \delta$ in the denominator, it actually has no singularity when $p \cdot \delta=0$. Thus, as stated there, this $(p \cdot \delta)^{-1}$ can be interpreted in any way one wishes. This is not true of the sum of the right-hand sides of (4.1) and (4.2). In other words, $I_{\mu \nu \sigma}^{T(1)}+I_{\mu \nu \sigma}^{T(2)}$ is singular when $p_{1} \cdot \delta=0$, or $p_{2} \cdot \delta=0$, or $p_{3} \cdot \delta=0$. If inappropriate meanings were assigned to these $(p \cdot \delta)^{-1}$, then the charge-conjugation invariance (4.9) would not be satisfied. In order to have (4.9), it is necessary to have

$$
\frac{1}{p \cdot \delta}=-\left.\frac{1}{p \cdot \delta}\right|_{\delta \rightarrow-\delta}
$$

where $p$ is any of the $p_{i}$. This is a non-trivial condition. Let $1 /(p \cdot \delta)$ be interpreted as

$$
\frac{1}{p \cdot \delta} \rightarrow a \frac{1}{p \cdot \delta+i \epsilon}+b \frac{1}{p \cdot \delta-i \epsilon}
$$


where $a+b=1$. Then

$$
-\left.\frac{1}{p \cdot \delta}\right|_{\delta \rightarrow-\delta} \rightarrow a \frac{1}{p \cdot \delta-i \epsilon}+b \frac{1}{p \cdot \delta+i \epsilon}
$$

It therefore follows from (4.10)-(4.12) that $a=b=\frac{1}{2}$, i.e., $1 /(p \cdot \delta)$ must be interpreted in the sense of a principal value,

$$
\begin{aligned}
\frac{1}{p \cdot \delta} & \rightarrow \frac{1}{2}\left(\frac{1}{p \cdot \delta+i \epsilon}+\frac{1}{p \cdot \delta-i \epsilon}\right) \\
& =\mathcal{P} \frac{1}{p \cdot \delta} .
\end{aligned}
$$

This answers question (A) of the introduction.

In Sec. 3, it has been found that the most general ee $\gamma \gamma$ vertex is given by (3.22). In this vertex, there is an arbitrary symmetric function $A\left(p_{1}, p_{2}\right)$. Suppose this general $e e \gamma \gamma$ vertex is used to calculate the present $\gamma \gamma \gamma$ amplitude, would this arbitrary $A\left(p_{1}, p_{2}\right)$ appear in the answer? If so, then the point-splitting regularization is not unique for quantum electrodynamics, i.e., there are many possible ways to apply this point-splitting regularization.

Fortunately this is not the case. The underlying reason is that the factor $\not p_{1}+\not p_{2}$ that multiplies $A\left(p_{1}, p_{2}\right)$ can be used, via (2.5), to cancel electron denominators, as already discussed immediately after (3.15). Thus the introduction of this $A\left(p_{1}, p_{2}\right)$ in the ee $\gamma \gamma$ vertex leads to an additional term in the $e e \gamma \gamma \gamma$ vertex. These extra terms cancel each other when all the external electron lines are on mass shell.

The $\gamma \gamma \gamma$ case of the present section gives an explicit demonstration of this cancellation. When the ee $\gamma \gamma$ vertex $V_{\mu \nu}^{(2)}\left(p_{1}, p_{2}\right)$ is used to treat the present case instead of (1.2), there are six diagrams: two triangle diagrams with three electron propagators, three diagrams with two electron propagators and one ee $\gamma \gamma$ vertex, and one diagram with one electron propagator and an $e e \gamma \gamma \gamma$ vertex. With (3.22) and following the procedure of Sec. 3, the most general ee $\gamma \gamma \gamma$ vertex is found to be given by

$$
\begin{gathered}
V_{\mu \nu \sigma}^{(3)}\left(p_{1}, p_{2}, p_{3}\right)=\frac{2 i e^{3}}{\left(p_{1} \cdot \delta\right)\left(p_{2} \cdot \delta\right)\left(p_{3} \cdot \delta\right)} \\
\times\left\{( p _ { 1 } \cdot \delta ) \left[\cos \left[\left(p_{2}-p_{3}\right) \cdot \delta\right]-\frac{1}{2} \cos \left[\left(p_{3}-p_{1}\right) \cdot \delta\right]-\frac{1}{2} \cos \left[\left(p_{1}-p_{2}\right) \cdot \delta\right]\right.\right. \\
\left.\quad-\sin \frac{\left(2 p_{1}-p_{2}-p_{3}\right) \cdot \delta}{2} A\left(p_{2}, p_{3}\right)\right] \gamma_{\mu} \delta_{\nu} \delta_{\sigma}
\end{gathered}
$$




$$
\begin{aligned}
& +\left(p_{2} \cdot \delta\right)\left[\cos \left[\left(p_{3}-p_{1}\right) \cdot \delta\right]-\frac{1}{2} \cos \left[\left(p_{1}-p_{2}\right) \cdot \delta\right]-\frac{1}{2} \cos \left[\left(p_{2}-p_{3}\right) \cdot \delta\right]\right. \\
& \left.-\sin \frac{\left(2 p_{2}-p_{3}-p_{1}\right) \cdot \delta}{2} A\left(p_{3}, p_{1}\right)\right] \delta_{\mu} \gamma_{\nu} \delta_{\sigma} \\
& +\left(p_{3} \cdot \delta\right)\left[\cos \left[\left(p_{1}-p_{2}\right) \cdot \delta\right]-\frac{1}{2} \cos \left[\left(p_{2}-p_{3}\right) \cdot \delta\right]-\frac{1}{2} \cos \left[\left(p_{3}-p_{1}\right) \cdot \delta\right]\right. \\
& \left.-\sin \frac{\left(2 p_{3}-p_{1}-p_{2}\right) \cdot \delta}{2} A\left(p_{1}, p_{2}\right)\right] \delta_{\mu} \delta_{\nu} \gamma_{\sigma} \\
& -\delta_{\mu} \delta_{\nu} \delta_{\sigma}\left[\not p_{1} \cos \left[\left(p_{2}-p_{3}\right) \cdot \delta\right]+\not p_{2} \cos \left[\left(p_{3}-p_{1}\right) \cdot \delta\right]+\not p_{3} \cos \left[\left(p_{1}-p_{2}\right) \cdot \delta\right]\right. \\
& \left.\left.-B\left(p_{1}, p_{2}, p_{3}\right)\left(p_{1}+\not p_{2}+\not p_{3}\right)\right]\right\} \text {. }
\end{aligned}
$$

Note the appearance of the three $A^{\prime} s$ and also an additional arbitrary symmetrical function $B\left(p_{1}, p_{2}, p_{3}\right)$. These three $A^{\prime} s$ cancel those coming from the three diagrams with an $e e \gamma \gamma$ vertex each. In the present case, analogous to the situation with the photon self-energy of Sec. 3, the new arbitrary function $B\left(p_{1}, p_{2}, p_{3}\right)$ does not contribute because $p_{1}+p_{2}+p_{3}=0$.

The independence on these arbitrary functions, coming from the cancellation of electron denominators, implies that there is a unique prescription for point-splitting regularization for every given value of $\delta_{\mu}$. Thus question (B) of the introduction is answered in the affirmative. Furthermore, this unique prescription is extremely simple as given by (1.2).

\section{Discussion}

We have seen that, given a four-vector $\delta_{\mu}$, the Feynman rule with point-splitting distance $\delta_{\mu}$ is unique and extremely simple; it is already given in the abstract together with the principal-value prescription (4.13). The following comments are perhaps useful.

(A) The present Feynman rule for point splitting is not equivalent to the one used previously [1]. This is the explanation for the peculiarity in [1], where, while gauge invariance is exactly satisfied for the one-loop photon self-energy, it holds for photonphoton scattering only for small external momenta. With the present Feynman rule, gauge invariance of course holds in all cases. On the other hand, this change from the previous treatment does not affect the calculation and results of [2] and [3]. In particular, the mass formulas (1.1) remain the same.

(B) As stated previously, there are two equivalent formulations of point splitting regularization. The simpler of the two approaches is to apply the Feynman rule (1.2) to each vertex. The alternative is to delete the second term in (1.2), but to introduce 
additional vertices ee $\gamma \gamma, e e \gamma \gamma \gamma$, etc. in such a way as to restore gauge invariance. The advantage of this otherwise clumsy procedure is that the Feynman rule (1.2) can be shown to be unique.

The Feynman rule for the eer $\gamma$ vertex is given by (3.22), and the rule for the ee $\gamma \gamma \gamma$ vertex is given by (4.14). For the sake of completeness, the ee $\gamma \gamma \gamma \gamma$ vertex is written down explicitly in the Appendix. Its complexity is in sharp contrast to to the simplicity of (1.2).

(C) Under some circumstances, double poles of the form $(p \cdot \delta)^{-2}$ can occur. For example, they appear in the calculations of cross sections through the squares of matrix elements. They can also appear in the matrix element itself when there is an internal photon line to be integrated over. A simple example is provided by the two-loop photon self-energy. In this case, it has been carefully checked that a $(p \cdot \delta)^{-2}$ does appear in the sense that the coefficient of this $(p \cdot \delta)^{-2}$ term does not vanish when $p \cdot \delta=0$. Here $p$ is the momentum of the internal photon line.

In such cases, the $(p \cdot \delta)^{-2}$ must be interpreted in the same way as (4.13), namely

$$
\frac{1}{(p \cdot \delta)^{2}}=-\frac{\partial}{\partial(p \cdot \delta)} \frac{1}{p \cdot \delta} \rightarrow-\frac{\partial}{\partial(p \cdot \delta)} \mathcal{P} \frac{1}{p \cdot \delta} .
$$

(D) For our program of determining theoretically the masses of the Higgs boson and the top quark [1]-[3], the next step is to obtain radiative corrections to (1.1). Since the first mass relation [9], [2], from terms of the order of $\delta^{-2}$, is the simpler one, it seems reasonable to begin with the two-loop Higgs tadpole. This radiative correction should give some indication of the accuracy of the relations (1.1). There are 166 diagrams for the two-loop Higgs tadpole. Because of this rather large number, the simplicity of the new Feynman rule here, together with the absence of additional vertices, is expected to be of central importance.

\section{Appendix A}

The results eqs. (3.22) for two photons and (4.14) for three photons can be generalized iteratively to any number of photons. For completeness we give in this Appendix the case of four photons:

$$
\begin{array}{r}
V_{\mu \nu \sigma \rho}^{(4)}\left(p_{1}, p_{2}, p_{3}, p_{4}\right)=C_{1}\left(p_{1} ; p_{2}, p_{3}, p_{4}\right) \gamma_{\mu} \delta_{\nu} \delta_{\sigma} \delta_{\rho}+C_{1}\left(p_{2} ; p_{3}, p_{4}, p_{1}\right) \delta_{\mu} \gamma_{\nu} \delta_{\sigma} \delta_{\rho} \\
+C_{1}\left(p_{3} ; p_{4}, p_{1}, p_{2}\right) \delta_{\mu} \delta_{\nu} \gamma_{\sigma} \delta_{\rho}+C_{1}\left(p_{4} ; p_{1}, p_{2}, p_{3}\right) \delta_{\mu} \delta_{\nu} \delta_{\sigma} \gamma_{\rho} \\
+\delta_{\mu} \delta_{\nu} \delta_{\sigma} \delta_{\rho}\left[C_{2}\left(p_{1} ; p_{2}, p_{3}, p_{4}\right) p_{1}+C_{2}\left(p_{2} ; p_{3}, p_{4}, p_{1}\right) p_{2}\right. \\
\left.+C_{2}\left(p_{3} ; p_{4}, p_{1}, p_{2}\right) p_{3}+C_{2}\left(p_{4} ; p_{1}, p_{2}, p_{3}\right) p_{4}\right]
\end{array}
$$


where

$$
\begin{aligned}
C_{1}\left(p_{1} ; p_{2}, p_{3}, p_{4}\right)= & \frac{e^{4}}{\left(p_{2} \cdot \delta\right)\left(p_{3} \cdot \delta\right)\left(p_{4} \cdot \delta\right)} \\
\times & \left\{\sin \left[\frac{1}{2}\left(3 p_{1}-3 p_{2}+p_{3}-p_{4}\right) \cdot \delta\right]+\sin \left[\frac{1}{2}\left(3 p_{1}-3 p_{2}-p_{3}+p_{4}\right) \cdot \delta\right]\right. \\
+ & \sin \left[\frac{1}{2}\left(3 p_{1}+p_{2}-3 p_{3}-p_{4}\right) \cdot \delta\right]+\sin \left[\frac{1}{2}\left(3 p_{1}-p_{2}-3 p_{3}+p_{4}\right) \cdot \delta\right] \\
+ & \sin \left[\frac{1}{2}\left(3 p_{1}+p_{2}-p_{3}-3 p_{4}\right) \cdot \delta\right]+\sin \left[\frac{1}{2}\left(3 p_{1}-p_{2}+p_{3}-3 p_{4}\right) \cdot \delta\right] \\
- & \sin \left[\frac{1}{2}\left(p_{1}-p_{2}+3 p_{3}-3 p_{4}\right) \cdot \delta\right]-\sin \left[\frac{1}{2}\left(p_{1}-p_{2}-3 p_{3}+3 p_{4}\right) \cdot \delta\right] \\
- & \sin \left[\frac{1}{2}\left(p_{1}-3 p_{2}-p_{3}+3 p_{4}\right) \cdot \delta\right]-\sin \left[\frac{1}{2}\left(p_{1}+3 p_{2}-p_{3}-3 p_{4}\right) \cdot \delta\right] \\
- & \sin \left[\frac{1}{2}\left(p_{1}+3 p_{2}-3 p_{3}-p_{4}\right) \cdot \delta\right]-\sin \left[\frac{1}{2}\left(p_{1}-3 p_{2}+3 p_{3}-p_{4}\right) \cdot \delta\right] \\
& +2\left[\cos \left[\frac{1}{2}\left(p_{1}+3 p_{2}-2 p_{3}-2 p_{4}\right) \cdot \delta\right]-\cos \left[\frac{1}{2}\left(3 p_{1}+p_{2}-2 p_{3}-2 p_{4}\right) \cdot \delta\right]\right] A\left(p_{3}, p_{4}\right) \\
& +2\left[\cos \left[\frac{1}{2}\left(p_{1}-2 p_{2}+3 p_{3}-2 p_{4}\right) \cdot \delta\right]-\cos \left[\frac{1}{2}\left(3 p_{1}-2 p_{2}+p_{3}-2 p_{4}\right) \cdot \delta\right]\right] A\left(p_{4}, p_{2}\right) \\
& +2\left[\cos \left[\frac{1}{2}\left(p_{1}-2 p_{2}-2 p_{3}+3 p_{4}\right) \cdot \delta\right]-\cos \left[\frac{1}{2}\left(3 p_{1}-2 p_{2}-2 p_{3}+p_{4}\right) \cdot \delta\right]\right] A\left(p_{2}, p_{3}\right) \\
& \left.-4 \sin \left[\frac{1}{2}\left(3 p_{1}-p_{2}-p_{3}-p_{4}\right) \cdot \delta\right] B\left(p_{2}, p_{3}, p_{4}\right)\right\},
\end{aligned}
$$

and

$$
\begin{aligned}
C_{2}\left(p_{1} ; p_{2}, p_{3}, p_{4}\right) & =\frac{e^{4}}{\left(p_{1} \cdot \delta\right)\left(p_{2} \cdot \delta\right)\left(p_{3} \cdot \delta\right)\left(p_{4} \cdot \delta\right)} \\
\times\{- & \frac{1}{4} \sin \left[\frac{1}{2}\left(3 p_{1}-3 p_{2}+p_{3}-p_{4}\right) \cdot \delta\right]+\sin \left[\frac{1}{2}\left(3 p_{1}-3 p_{2}-p_{3}+p_{4}\right) \cdot \delta\right] \\
& +\sin \left[\frac{1}{2}\left(3 p_{1}+p_{2}-3 p_{3}-p_{4}\right) \cdot \delta\right]+\sin \left[\frac{1}{2}\left(3 p_{1}-p_{2}-3 p_{3}+p_{4}\right) \cdot \delta\right] \\
& +\sin \left[\frac{1}{2}\left(3 p_{1}+p_{2}-p_{3}-3 p_{4}\right) \cdot \delta\right]+\sin \left[\frac{1}{2}\left(3 p_{1}-p_{2}+p_{3}-3 p_{4}\right) \cdot \delta\right] \\
- & 3 \sin \left[\frac{1}{2}\left(p_{1}-p_{2}+3 p_{3}-3 p_{4}\right) \cdot \delta\right]-3 \sin \left[\frac{1}{2}\left(p_{1}-p_{2}-3 p_{3}+3 p_{4}\right) \cdot \delta\right] \\
- & 3 \sin \left[\frac{1}{2}\left(p_{1}-3 p_{2}-p_{3}+3 p_{4}\right) \cdot \delta\right]-3 \sin \left[\frac{1}{2}\left(p_{1}+3 p_{2}-p_{3}-3 p_{4}\right) \cdot \delta\right] \\
- & \left.3 \sin \left[\frac{1}{2}\left(p_{1}+3 p_{2}-3 p_{3}-p_{4}\right) \cdot \delta\right]-3 \sin \left[\frac{1}{2}\left(p_{1}-3 p_{2}+3 p_{3}-p_{4}\right) \cdot \delta\right]\right] \\
- & {\left[\cos \left[\frac{1}{2}\left(p_{1}+3 p_{2}-2 p_{3}-2 p_{4}\right) \cdot \delta\right]-\cos \left[\frac{1}{2}\left(3 p_{1}+p_{2}-2 p_{3}-2 p_{4}\right) \cdot \delta\right]\right] A\left(p_{3}, p_{4}\right) } \\
- & {\left[\cos \left[\frac{1}{2}\left(p_{1}-2 p_{2}+3 p_{3}-2 p_{4}\right) \cdot \delta\right]-\cos \left[\frac{1}{2}\left(3 p_{1}-2 p_{2}+p_{3}-2 p_{4}\right) \cdot \delta\right]\right] A\left(p_{4}, p_{2}\right) } \\
& -\left[\cos \left[\frac{1}{2}\left(p_{1}-2 p_{2}-2 p_{3}+3 p_{4}\right) \cdot \delta\right]-\cos \left[\frac{1}{2}\left(3 p_{1}-2 p_{2}-2 p_{3}+p_{4}\right) \cdot \delta\right]\right] A\left(p_{2}, p_{3}\right) \\
& \quad-\sin \left[\frac{1}{2}\left(p_{1}+p_{2}-p_{3}-p_{4}\right) \cdot \delta\right] A\left(p_{1}, p_{2}\right) A\left(p_{3}, p_{4}\right) \\
& -\sin \left[\frac{1}{2}\left(p_{1}-p_{2}+p_{3}-p_{4}\right) \cdot \delta\right] A\left(p_{1}, p_{3}\right) A\left(p_{2}, p_{4}\right) \\
& \left.-\sin \left[\frac{1}{2}\left(p_{1}-p_{2}-p_{3}+p_{4}\right) \cdot \delta\right] A\left(p_{1}, p_{4}\right) A\left(p_{2}, p_{3}\right)+C\left(p_{1}, p_{2}, p_{3}, p_{4}\right)\right\} \cdot(\mathrm{A} .3)
\end{aligned}
$$




\section{Acknowledgments}

For many discussions, we are grateful to Professor Raymond Gastmans and Professor Maurice Jacob. One of us (TTW) thanks Dr. Lia Calin for an idea of checking gauge

invariance, and he and $\mathrm{CN}$ wish to thank the Theory Division of CERN for its kind hospitality. 


\section{References}

[1] P. Osland and T. T. Wu, Zeitschrift f. Physik C 55 (1992) 569.

[2] P. Osland and T. T. Wu, Zeitschrift f. Physik C 55 (1992) 585.

[3] P. Osland and T. T. Wu, Zeitschrift f. Physik C 55 (1992) 593.

[4] S. L. Glashow, Nucl. Phys. 22 (1961) 579;

S. Weinberg, Phys. Rev. Lett. 19 (1967) 1264;

A. Salam, in Elementary Particle Theory: Relativistic Groups and Analyticity (Nobel Symposium No. 8), ed. N. Svartholm (Almqvist and Wiksell, Stockholm, 1968), p. 367 ;

S. L. Glashow, J. Iliopoulos, and L. Maiani, Phys. Rev. D2 (1970) 1285.

[5] F. Englert and R. Brout, Phys. Rev. Lett. 13 (1964) 321;

P. W. Higgs, Phys. Lett. 12 (1964) 132; Phys. Rev. Lett. 13 (1964) 508;

G. S. Guralnik, C. R. Hagen and T. W. B. Kibble, Phys. Rev. Lett. 13 (1964) 585;

P. W. Higgs, Phys. Rev. 145 (1966) 1156;

T. W. B. Kibble, Phys. Rev. 155 (1967) 1554.

[6] P. A. M. Dirac, Proc. Cambridge Phil. Soc. 30 (1934) 150;

R. Peierls, Proc. Roy. Soc., Series A 146 (1934) 420;

J. Schwinger, Phys. Rev. 82 (1951) 664;

K. Johnson, in Lectures on Particles and Field Theory, Lectures at Brandeis University 1964, eds. S. Deser and K. W. Ford, vol. 2 (Prentice-Hall, Inc., 1965) p. 1

[7] P. Osland and T. T. Wu, Phys. Lett. B 291 (1992) 315.

[8] W. H. Furry, Phys. Rev. 51 (1937) 125.

[9] R. Decker and J. Pestieau, Preprint UCL-IPT-79-19, Université de Louvain (1979); DESY Workshop, October 22-24, 1979;

M. Veltman, Acta Physica Polonica B12 (1981) 437. 


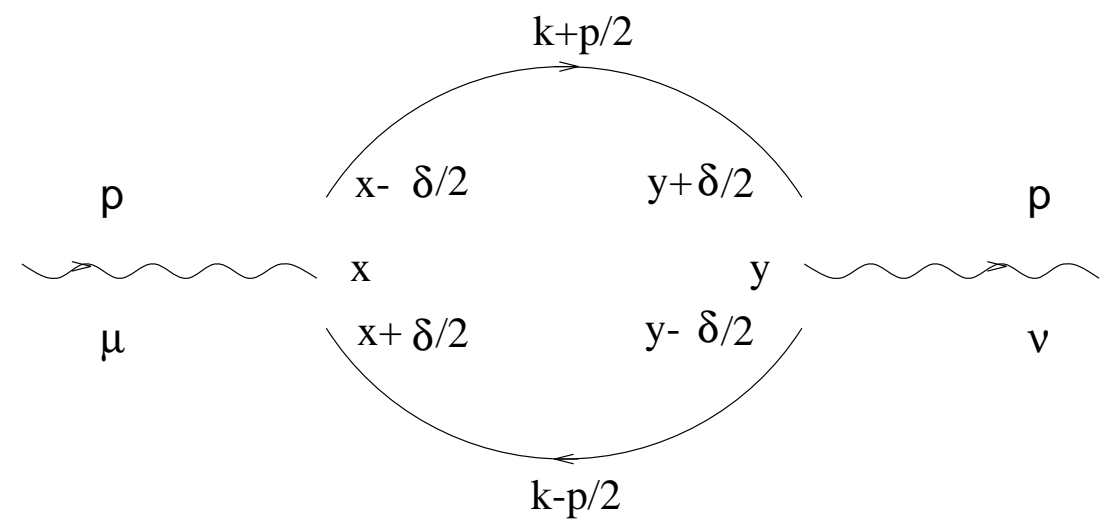

Figure 1

Fig. 1. One-loop contribution to the photon self-energy. 

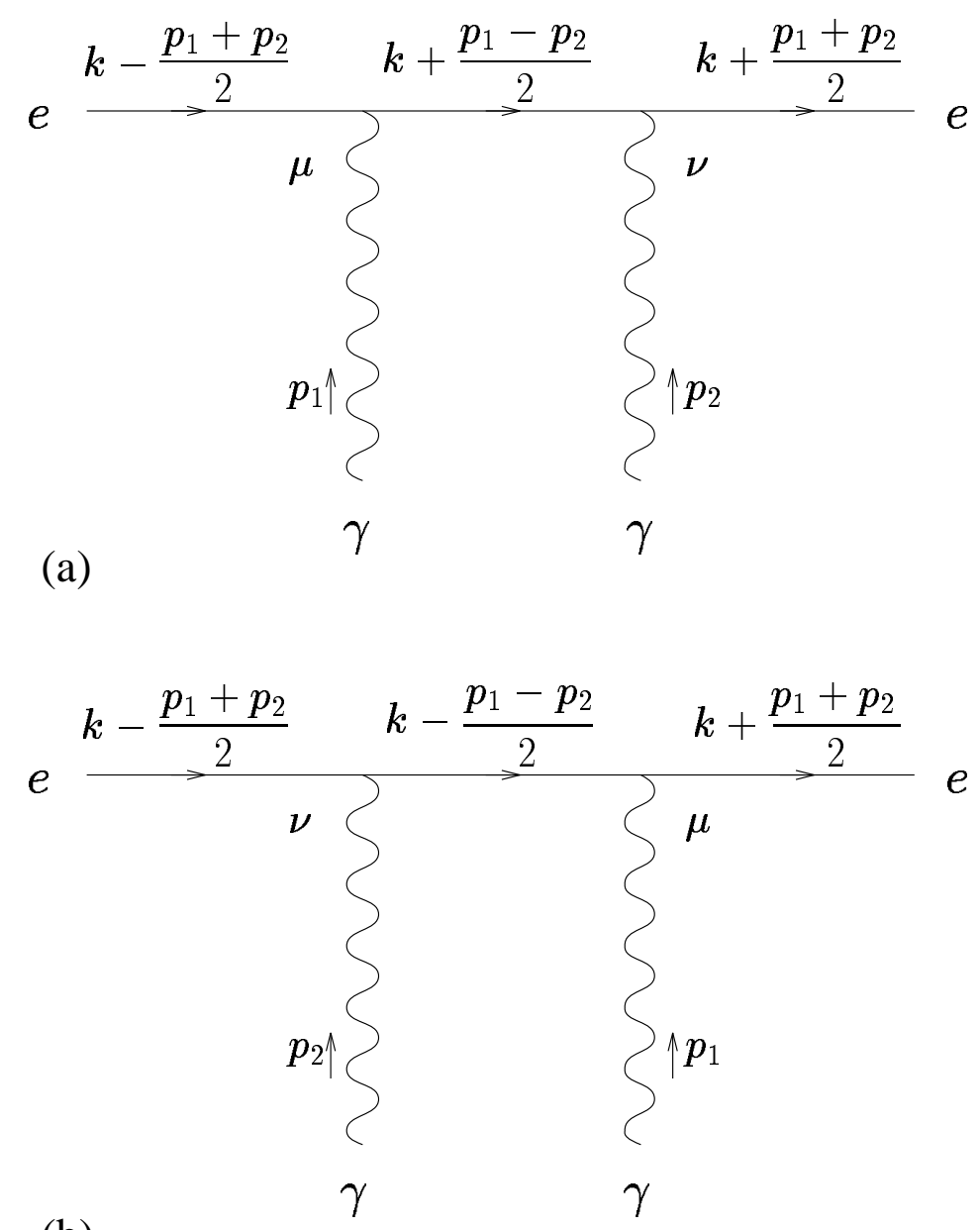

Figure 2

Fig. 2. Two Feynman diagrams for the verification of gauge invariance in the presence of point splitting. The external lines are not on mass shell. 


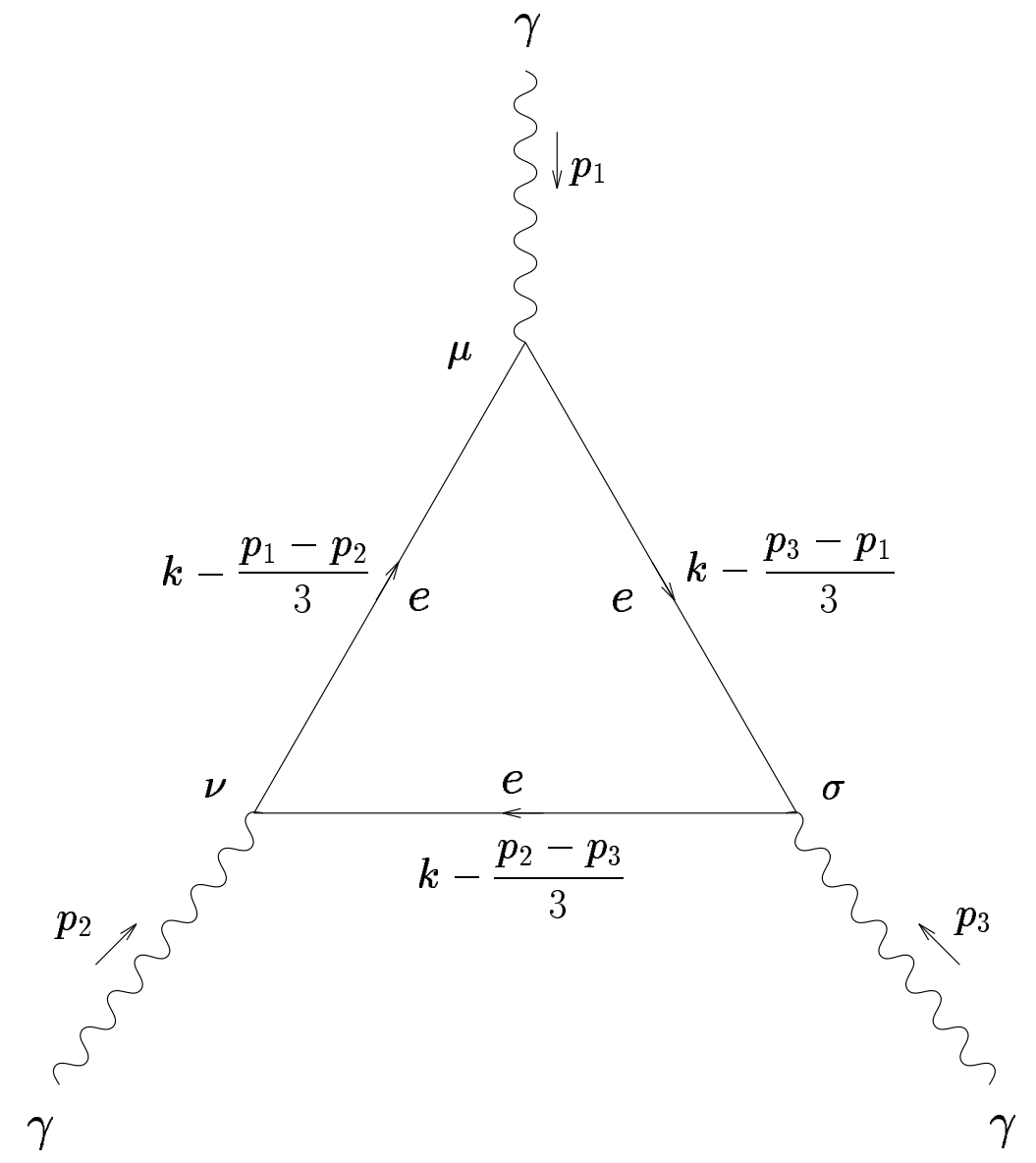

Figure 3

Fig. 3. One of the two one-loop contributions to the three-photon vertex. The other diagram differs from this one in having the direction of the electron line reversed. 
